\title{
Redonnons leur importance aux soins bucco-dentaires
}

$\mathrm{L}$ es soins bucco-dentaires constituent une activité économique importante au Canada et représentent $7 \%$ du total des dépenses consacrées aux soins de santé. Le coût des soins dispensés par les dentistes et autres membres des équipes de santé bucco-dentaire a atteint environ 9,7 milliards de dollars en $2005^{1}$. Ce montant ne devrait pas nous étonner, étant donné que la carie dentaire constitue la maladie chronique la plus répandue chez les enfants du Canada. De plus, beaucoup de lecteurs du journal sont susceptibles d'être atteints à un degré ou un autre de parodontopathie, principale cause de perte des dents chez l'adulte.

En dépit de ces vérités, il est rarement question de santé bucco-dentaire et de soins dentaires dans les rapports sur le système de santé du Canada publiés au cours des dernières années. La lecture de ces documents donne l'impression que pour les responsables des politiques sur la santé, la bouche ne fait pas partie du corps. C'est peut-être parce que l'art dentaire s'est créé au Canada un système de prestation de soins en grande partie privé (95\% dans le secteur privé) qui fonctionne essentiellement dans un domaine différent de la profession médicale.

Nos deux professions affichent un fier bilan de collaboration bénéfique. Par exemple, nous avons collaboré de près dans des questions d'intérêt public, comme la fluoruration de l'eau et l'éducation des professionnels. Je crois toutefois que le moment est maintenant bien choisi pour redoubler nos efforts conjoints pour le bien public, parce que les systèmes de santé sont de plus en plus intégrés et que la santé bucco-dentaire devient de plus en plus importante pour le public.

Cette importance accrue émane de recherches qui démontrent l'existence d'un lien entre les maladies bucco-dentaires et certains problèmes de santé systémiques coûteux ${ }^{2}$. Les liens semblent très solides dans le cas du diabète et des pneumonies d'inhalation, mais plus ténus pour le moment dans le cas de la cardiopathie ischémique et des accouchements prématurés. L'existence possible de ces liens intéresse les décideurs de certains milieux assez pour qu'ils encouragent à pousser plus loin la recherche sur la question.

Comme il est déjà clair que les maladies bucco-dentaires et les principales maladies chroniques qui mettent la vie en danger présentent des facteurs de risque communs comme le tabagisme et l'apport non contrôlé de glucides, nous devons commencer à envisager de sortir du cadre courant du financement de la santé.

C'est particulièrement important à une époque où le public et les décideurs s'intéressent à la prévention des maladies et à la promotion de la santé. Une des grandes réussites de notre profession - dentaire axée sur la prévention, c'est le recul spectaculaire de la prévalence des maladies bucco-dentaires chez beaucoup de Canadiens depuis le début des années 1970, époque à laquelle remonte notre dernière enquête nationale sur la santé bucco-dentaire.

Même si nous n'avons pas de données épidémiologiques récentes, je suis convaincu que les groupes plus vulnérables de notre population, comme les personnes désavantagées sur le plan économique, les Autochtones, les personnes handicapées et les personnes âgées, portent une partie injuste du fardeau des maladies bucco-dentaires ${ }^{3}$. Ce sont malheureusement les groupes les moins capables d'avoir accès aux soins de santé bucco-dentaire complets mis à la disposition de la majorité des Canadiens.

Il y a toutefois des nouvelles encourageantes qui s'annoncent. Comme on le recommande dans la Stratégie canadienne de santé bucco-dentaire ${ }^{3}$, le travail de terrain est déjà commencé pour lancer une enquête nationale sur la santé bucco-dentaire dans le cadre de l'Enquête canadienne sur les mesures de la santét. Je crois que l'information réunie grâce à cet exercice fera comprendre que la prévention des maladies bucco-dentaires chez les groupes vulnérables est souhaitable sur le plan social et logique sur le plan économique pour le système de santé public du Canada.

Nos deux professions ont certainement l'obligation conjointe de préconiser qu'on affecte davantage de ressources (humaines et financières) à la recherche sur la prévention des maladies buccodentaires et sur les liens entre celles-ci et les maladies systémiques. Même si les soins de santé bucco-dentaire absorbent des ressources financières importantes au Canada, la proportion du budget des Instituts de recherche en santé du Canada qui est consacrée à la recherche en la matière, principalement par l'entremise de l'Institut de l'appareil locomoteur et de l'arthrite, ne reflète pas adéquatement le fardeau sanitaire imposé par les maladies bucco-dentaires.

Au moment où nos facultés de médecine et d'art dentaire se rapprochent, les chercheurs en santé bucco-dentaire collaborent maintenant davantage avec ceux d'autres disciplines. La bouche est le point d'accès à beaucoup des systèmes corporels. Les médecins et les dentistes doivent se consulter plus souvent pour régler des problèmes cliniques d'intérêt commun. Le moment est venu de reconnaître que la bouche n'est pas séparée du reste du corps. J'exhorte les professions médicale et dentaire à conjuguer leurs efforts pour encourager le système de santé du Canada en pleine évolution à redonner son importance à la bouche.

\section{Wayne Halstrom \\ Président, Association dentaire canadienne Ottawa (Ont.)}

Cet article a fait l'objet d'un examen par les pairs.

\section{RÉFÉRENCES}

I. Institut canadien d'information sur la santé. Tendances des dépenses nationales de santé, 1975-2005. Ottawa : Institut canadien d'information sur la santé; 2005.

2. Département de la Santé et des Services humains des États-Unis; National Institute of Dental and Craniofacial Research. Oral health in America: a report of the Surgeon General. Septembre 2000. Disponible à : www2.nidcr.nih.gov/sgr/sgrohweb /welcome.htm (consulté le 23 novembre 2006).

3. Federal P, Directors TD. Une stratégie canadienne de santé bucco-dentaire. Disponible : www.fptdd.ca/COHSdoc.html (consulté le 23 novembre 2006).

4. Statistique Canada. Enquête canadienne sur les mesures de la santé. Disponible à : www.statcan.ca/francais/concepts/hs/measures_f.htm (consulté le 23 novembre 2006). 